\title{
On the fractal characteristics of the $\eta$ model
}

\author{
Angel Sánchez ${ }^{\mathrm{a}, \mathrm{b}, 1}$, Francisco Guinea ${ }^{\mathrm{c}, \mathrm{d}}$, Enrique Louis ${ }^{\mathrm{e}}$ \\ and Vincent Hakim ${ }^{\mathrm{f}}$ \\ ${ }^{2}$ Departamento de Física Teórica I, Facultad de Ciencias Físicas, Universidad Complutense, \\ E-28040 Madrid, Spain \\ ${ }^{\mathrm{h}}$ Theoretical Division and Center for Nonlinear Studies, Los Alamos National Laboratory, \\ Los Alamos, NM 87545, USA \\ "Instituto de Ciencia de Materiales, Facultad de Ciencias C-XII, Universidad Autónoma, \\ Canto Blanco, E-28049 Madrid, Spain \\ ${ }^{\mathrm{d}}$ The Harrison M. Randall Laboratory of Physics, The University of Michigan, Ann Arbor, \\ MI 48109-1120, USA \\ 'Departamento de Física Aplicada, Universidad de Alicante, Apartado 99, E-03080 Alicante, \\ Spain \\ ${ }^{\mathrm{f}}$ Laboratoire de Physique Statistique, ENS et Universités Paris VI et VII, 24 Rue Lhomond, \\ 75231 Paris Cedex 05, France
}

Since the $\eta$ or dielectric breakdown model was proposed, it has been generally accepted that the fractal characteristics of the so-grown clusters have a smooth behavior as $\eta$ increases from 0 to infinity. On the basis of recent theoretical calculations on a related model, we conjecture that the aggregate can become effectively branchless for $\eta$ larger than a critical value $\eta_{1}$. A related possibility is that the value 1 for the fractal dimension might be reached at finite values of $\eta$. We have carried out a large simulation program to test these conjectures and we find evidence supporting their validity. This is a preliminary report of our work on this problem.

\section{Introduction}

Among the models proposed in the last decade to describe different growth phenomena, the $\eta$ or dielectric breakdown (DB) model [1] is perhaps the one which shows the largest variety of interesting features. Besides, it has become extremely useful to understand not only the phenomenon of dielectric breakdown, but also a number of others like electrodeposition, fluid-fluid displacement, or fluid flow in porous media (see, e.g., ref. [2], and references therein). It is also closely related to models for dendritic growth and crack formation. Finally, it includes as particular realizations for certain values of the parameter $\eta$ other growth models, such as the Eden model [3] or the diffusion-limited

\footnotetext{
${ }^{1}$ Address after October 1st 1992: Escuela Politécnica Superior, Universidad Carlos III de Madrid, E-28913 Leganés, Madrid, Spain.
} 
aggregation (DLA) [4], and therefore it provides a way to study them in the more general context of Laplacian growth processes.

The $\eta$ model is defined [1] by a growth rule that assigns at any point of the surface of the evolving cluster a growth probability proportional to the local normal electric (Laplacian, in general) field raised to the power $\eta$, i.e., $P_{\eta}(x) \propto[n \cdot \nabla \Phi(x)]^{\eta}, x$ being a cluster surface point and $\Phi$ being the electric potential. The cluster is assumed to be equipotential $(\Phi=0)$ and far from it there is an enclosing circular electrode kept at constant potential $(\Phi=1)$. The resulting aggregates are branched structures, the complexity of the ramification decreasing with increasing $\eta$. For instance, if $\eta=0$, the growth probability is purely random and independent of the Laplacian field (and the model becomes the Eden one, as we announced above); the corresponding clusters are compact objects, although with a very complicated surface. When $\eta$ increases to 1 , more and more holes appear, defining branches in the clusters. For $\eta=1$, which from the definition can be seen to be the same as DLA, the well-known star-shaped aggregates arise.

Since the earliest simulations, an important parameter used to characterize the $\eta$ clusters was the fractal dimension in several of its forms, e.g., as obtained from the scaling of magnitudes such as the radius of gyration or the number of particles with the cluster size. The first values of the fractal dimension were computed in ref. [1], and it turned out that for $\eta=0,0.5,1$ and 2 the results were $D=2,1.89,1.75$ and 1.6 , respectively. Higher $\eta$ values were not analyzed, because the little ramification of the structure implied very large simulations to have reliable estimates of the fractal dimension. Later, Matsushita et al. derived [5] an expression for the dimension of the $\eta$ clusters, using a random-walk DLA-like version of the model (valid only for rational $\eta$ values). Their result, which indicated that $D \rightarrow 1$ continuously when $\eta \rightarrow \infty$, was also checked numerically [6], but, again, only up to $\eta=2$. Finally, motivated by theoretical work by Halsey [7], Amitrano carried out [8] a detailed numerical work, studying a large number of small (some 150 particles) clusters, grown with $\eta$ between 0 and 5 . Her results show a monotonic decreasing of $D$, and, for the largest $\eta$ value considered, $\eta=5, D=1.08$. This is, to our knowledge, the last result about this question.

\section{The needle model}

The reason to come back to the problem of the fractal dimension of the $\eta$ model and more generally to the behavior of the model fractal characteristics as $\eta$ increases is that, recently, Derrida and Hakim [9] have derived some results for a related model which suggest that the aggregate might become 
branchless for $\eta$ larger than a finite critical value $\eta_{1}$. This also raises the possibility that $D=1$ is reached for a finite value of $\eta$ which can coincide with $\eta_{1}$ or be larger. In this section, we summarize the results of ref. [9] and discuss their implications.

The overall branched structure of DLA and $\eta$ clusters can be simulated by a set of one-dimensional needles, growing from a central seed. In two dimensions, this model can be treated by conformal mapping techniques, and the field $\Phi$ calculated outside the aggregate. Once $\Phi$ is obtained, the growth probability and velocity are readily derived from it. Competition between needles of different sizes can be estimated, as function of their number and of $\boldsymbol{\eta}$. The most striking result of this analysis is the reduction of the number of stable needles to two, as $\eta \rightarrow 6$. Indeed, the stability condition for $n$ branches turns out to be

$$
\eta< \begin{cases}\frac{4}{n-2} & \text { for even } n, \\ \frac{4 n}{n^{2}-2 n-1} & \text { for odd } n .\end{cases}
$$

If this result for the needle model holds true for the $\eta$ model as well, the resulting aggregate should look effectively one-dimensional on a coarse-grained scale, and branching should be suppressed. The pattern could be modeled as a kind of random walk, with a statistical weight depending on its shape and on $\eta$. Such walks have already been discussed [10], under the name of Laplacian random walks. The possibility that for values of $\eta$ greater than 6 the aggregates generated by the $\mathrm{DB}$ model reduce to Laplacian random walks is rather intriguing, and opens new perspectives to study these growth processes.

\section{Simulation results and discussion}

In the present work we have generated a variety of aggregates for different values of $\eta$. The main difficulty in obtaining accurate statistics is the necessity of solving Laplace's equation in a sufficiently large lattice. We have used hexagonal lattices, to minimize anisotropy effects, of sizes up to $256 \times 256$. The smallest aggregates are those simulated for large values of $\eta$. They turned out to be close to a straight line, and reach soon the boundaries of the lattice. Typical sizes, for $\eta \geqslant 8$, are $\sim 300$. On the other hand, for typical DLA clusters $(\eta=1)$, aggregates with up to 20000 particles were generated. These sizes, which are significantly larger than previous calculations, appear to reach the limits of present computing capabilities. We are currently investigating alternative ways of studying these models. 


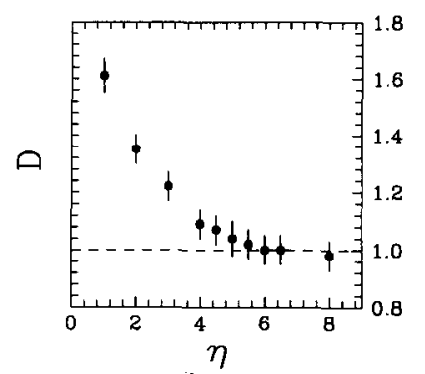

Fig. 1. Fractal (box-counting) dimension of the clusters as function of $\eta$. The dashed line indicates $D=1$.

Our main results are summarized in fig. 1. We present the fractal (boxcounting) dimension of the clusters generated, as function of $\eta$. For each $\eta$ value, an average of at least 3 clusters is presented. The fractal dimension, $D$, decreases as function of $\eta$, and becomes constant, around $D=1$, for $\eta \geqslant 6$. We have analyzed other magnitudes, like the mean number of particles per cluster and the mass-radius scaling dimension, and they also support the conjecture of the existence of a "transition". In particular, the mean number of particles per cluster is greater (smaller) than the lattice diameter for $\eta$ less (more) than 6 . This is a hint of a less intricated structure of the aggregates with $\eta \geqslant 6$, which would be related to the fact that they show $D=1$. Moreover, the number of branches of the grown clusters is in accordance with the aforementioned theoretical prediction of ref. [9]. For $\eta>2$ they never show more than three branches, and no presence of branches, on the scale of the aggregate size, can be observed for large values of $\eta$. Typical examples for $\eta=8$ are shown in fig. 2 (the side of the outcr hexagon is 256 ).
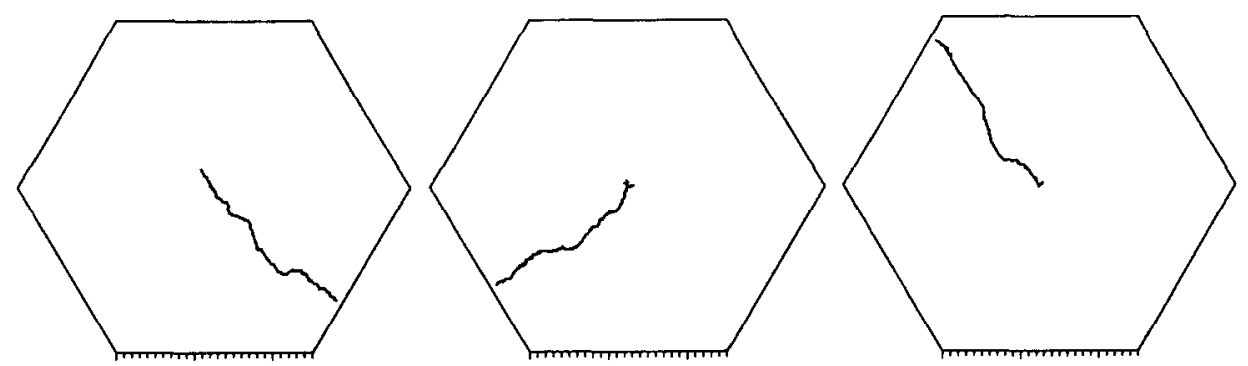

Fig. 2. Examples of $\eta=8$ clusters. Branches are practically absent and the clusters are ribbon-like. From left to right the number of particles is 319,304 and 288 , and their dimensions are $0.95 \pm 0.02$, $0.97 \pm 0.01$ and $0.96 \pm 0.02$, respectively. 


\section{Conclusion}

Our results support the hypothesis that the shape of the aggregates of the $\eta$ model changes into a ribbon-like object with no branches for $\eta \sim 6$. Moreover, it also seems that the resulting Laplacian random walk has a fractal dimension $D \sim 1$. This change in the behavior of the aggregates, which resembles a conventional phase transition in some aspects, opens new possibilities for the study of the model. The high $\eta$ regime, with its simple and quasi deterministic shapes, looks similar to the mean field solutions in statistical mechanics. It is interesting to check whether this analogy can be pushed further, and the deviations from unbranched objects can be understood within some kind of perturbation expansion. Further research along these lines, as well as to get a better characterization of the transition, is in progress.

\section{Acknowledgements}

We gratefully acknowledge permission from the Advanced Computing Laboratory of Los Alamos National Laboratory and the Universidad Complutense to use their computer facilities to grow the clusters studied in this work. A.S. was partially supported by the CICyT (Spain) project MAT90-0544, whereas the U.S. Department of Energy supported his work at Los Alamos. F.G. and E.L. were supported by CICyT (Spain).

\section{References}

[1] L. Niemeyer, L. Pietronero and H.J. Wiesmann, Phys. Rev. Lett. 52 (1984) 1033; L. Pietronero and H.J. Wiesmann, J. Stat. Phys. 36 (1984) 909.

[2] J. Feder, Fractals (Plenum, New York, 1988).

[3] M. Eden, in: Proc. Fourth Berkeley Symp. on Mathematical Statistics and Probability, vol. 4, J. Neyman, ed. (University of California Press, Berkeley, 1961) p. 223.

[4] T.A. Witten and L.M. Sander, Phys. Rev. Lett. 47 (1981) 1400.

[5] M. Matsushita, K. Honda, H. Toyoki, Y. Hayakawa and H. Kondo, J. Phys. Soc. Jpn. 55 (1986) 2618.

[6] Y. Hayakawa, H. Kondo and M. Matsushita, J. Phys. Soc. Jpn. 55 (1986) 2479.

[7] T. Halsey, Phys. Rev. Lett. 59 (1987) 2067.

[8] C. Amitrano, Phys. Rev. A 39 (1989) 6618.

[9] B. Derrida and V. Hakim, Phys. Rev. A 45 (1992) 8579.

[10] J.W. Lyklema, C. Everstz and L. Pietronero, Europhys. Lett. 2 (1986) 77; J.W. Lyklema and C. Everstz, J. Phys. A 19 (1986) L895; G.F. Lawler, J. Phys. A 20 (1986) 4656. 